\title{
Age-Related Decline in Circadian Output
}

\author{
Takahiro J. Nakamura, ${ }^{1,2}$ Wataru Nakamura, ${ }^{3}$ Shin Yamazaki, ${ }^{4}$ Takashi Kudo, ${ }^{1}$ Tamara Cutler, ${ }^{1}$ Christopher S. Colwell, ${ }^{1}$ \\ and Gene D. Block ${ }^{1}$ \\ ${ }^{1}$ Department of Psychiatry and Biobehavioral Sciences, University of California, Los Angeles, Los Angeles, California 90024-1759, ${ }^{2} \mathrm{Faculty}$ of \\ Pharmaceutical Sciences, Teikyo Heisei University, Ichihara, Chiba 290-0193, Japan, ${ }^{3}$ Laboratory of Oral Chronobiology, Graduate School of Dentistry, \\ Osaka University, Suita, Osaka 565-0871, Japan, and ${ }^{4}$ Department of Biological Sciences, Vanderbilt University, Nashville, Tennessee $37235-1634$
}

Disruptions in sleep/wake cycles, including decreased amplitude of rhythmic behaviors and fragmentation of the sleep episodes, are commonly associated with aging in humans and other mammals. While there are undoubtedly many factors contributing to these changes, a body of literature is emerging, suggesting that an age-related decline in the central circadian clock in the suprachiasmatic nucleus (SCN) may be a key element responsible. To explore age-related changes in the $\mathrm{SCN}$, we have performed in vivo multiunit neural activity (MUA) recordings from the SCN of freely moving young (3-5 months) and middle-aged (13-18 months) mice. Importantly, the amplitude of day-night difference in MUA was significantly reduced in the older mice. We also found that the neural activity rhythms are clearly degraded in the subparaventricular zone, one of the main neural outputs of the SCN. Surprisingly, parallel studies indicate that the molecular clockwork in the SCN as measured by PER2 exhibited only minor deficits at this same age. Thus, the circadian output measured at the level of neural activity rhythms in the SCN is degraded by aging, and this decline occurs before the disruption of key components of the molecular clockwork.

\section{Introduction}

Disruptions in sleep/wake cycles, including decreased amplitude of rhythmic behaviors and fragmentation of the sleep episodes, are commonly associated with aging in humans and other mammals (Bliwise, 1993; Turek et al., 1995; Dijk et al., 1999; Van Someren, 2000). The mechanisms underlying these changes are unknown; however, there is growing evidence that age-related changes within the central circadian clock (SCN) may be responsible. The aged SCN exhibits altered expression of the key neuropeptides (Kawakami et al., 1997; Krajnak et al., 1998; Duncan et al., 2001) that would be expected to disrupt the synchrony of the SCN population and decreases in glucose utilization (Wise et al., 1988) consistent with such a decrease in synchrony. The observation that some of the age-related decline in behavioral rhythmicity can be reversed by implanting fetal SCN tissue into the third ventricle (Van Reeth et al., 1994; Viswanathan and Davis, 1995; Cai et al., 1997) leads additional support to the hypothesis that age-related changes in the SCN play an important role in driving the age-related behavioral changes. Previous work has shown the electrophysiological activity of aged SCN neurons in vitro is altered (Satinoff et al., 1993; Watanabe et al., 1995; Aujard et al., 2001; Biello, 2009), findings that raise the possibility that electrical activity rhythms that are essential for circadian output

Received Jan. 26, 2011; revised May 23, 2011; accepted June 7, 2011.

Author contributions: T.J.N., W.N., S.Y., T.K., C.S.C., and G.D.B. designed research; T.J.N., W.N., T.K., and T.C. performed research; T.J.N. and W.N. analyzed data; C.S.C. and G.D.B. wrote the paper.

This work was supported by National Institutes of Health Grants R01 MH062517 (G.D.B.), P30-AG028748 (T.J.N.), and CHDI A-2702 (C.S.C.)

The authors declare no competing financial interests.

Correspondence should be addressed to Christopher S. Colwell, Department of Psychiatry and Biobehavioral Sciences, University of California, Los Angeles, 760 Westwood Boulevard, Semel Institute, Los Angeles, CA 90024-1759. E-mail: ccolwell@mednet.ucla.edu.

DOI:10.1523/JNEUROSCI.0451-11.2011

Copyright $\odot 2011$ the authors $\quad 0270-6474 / 11 / 3110201-05 \$ 15.00 / 0$ may be impacted early in the aging process. In the present study, we sought to determine whether circadian rhythms in spontaneous electrical firing in the SCN and subparaventricular zone $(\mathrm{SPZ})$ are reduced in freely behaving, middle-age mice.

\section{Materials and Methods}

Animals. Male C57BL/6J mice and PER2::LUC knock-in mice (C57BL/6J background) (Yoo et al., 2004) from our breeding colony were used for the experiment. Animals were maintained under controlled environmental conditions (temperature, $22 \pm 2^{\circ} \mathrm{C}$; light:dark [LD] 12:12 h, light $\sim 300$ lux) with food and water available ad libitum. All procedures and standards of care were approved by the University of California, Los Angeles Division of Laboratory Animals and were conducted according to the National Institutes of Health guidelines for the use of experimental animals.

Locomotor activity recording. Male C57BL/6J mice were classified into two groups: young adult (average age, $3.19 \pm 0.12$ months) and middleaged (12.75 \pm 0.21 months). Animals were then housed individually in cages with a running wheel. Locomotor activity was measured as running wheel revolutions recorded in $1 \mathrm{~min}$ bins and analyzed with CLOCKLAB software (Actimetrics).

In vivo multiunit neural activity recording from SCN and SPZ. The experiments were performed as previously described (Nakamura et al., $2008)$. Young adult ( $4.28 \pm 0.50$ months) and middle-aged (15.30 \pm 1.89 months) male C57BL/6J mice were anesthetized under isoflurane (2-5\%; Phoenix Pharmaceutical) and placed on a stereotaxic instrument (David Kopf Instruments). Electrodes were inserted into the brain, aimed at the SCN $(0.1 \mathrm{~mm}$ posterior and $0.2 \mathrm{~mm}$ lateral to the bregma, $5.9 \mathrm{~mm}$ depth from the skull surface) or SPZ ( $0.2 \mathrm{~mm}$ posterior, $0.2 \mathrm{~mm}$ lateral to the bregma, and $5.7 \mathrm{~mm}$ depth), and attached directly to the skull. After surgery, each mouse was transferred to an open-top cage with a running wheel mounted on one side in a light-tight box with a 12:12 h LD cycle. Output signals were processed by differential input integration amplifiers (Burr-Brown) and then fed into AC amplifiers (bandpass, $500 \mathrm{~Hz}$ to 5 $\mathrm{kHZ}$; gain, $\times 10,000)$. Spikes were discriminated by amplitude and counted in $1 \mathrm{~min}$ bins by using a computer-based window discrimination system. Simultaneously with neural activity monitoring, the loco- 
motor activity in individual mice also was detected as running wheel revolutions recorded in $1 \mathrm{~min}$ bins and stored on a computer. After study completion, each animal was anesthetized, and a positive current $(2 \mu \mathrm{A} ; 60 \mathrm{~s})$ was passed through the recording electrodes. The brain was removed and fixed in $4 \%$ paraformaldehyde in $0.1 \mathrm{~m}$ phosphate buffer containing $5 \%$ potassium ferrocyanide (Sigma-Aldrich). Serial coronal sections $(40 \mu \mathrm{m})$ were stained with neutral-red, and blue spots of deposited iron were identified with the recording site.

Immunohistochemistry. The methods were similar to those previously described (Kudo et al., 2011). Young adult (2-4 months; average age, $2.90 \pm 0.19$ months) and middle-aged (12 months; $11.96 \pm 0.07$ months) male C57BL/6J mice were used. Rabbit anti-PER2 (1:1000; Alpha Diagnostics) was used. All immunopositive cells within the SCN of these regions were counted manually at $400 \times$ with the aid of a grid $(200 \times 400 \mu \mathrm{m})$. All immunopositive cells within the grid were counted equally without regard to the intensity of the staining. Counts were performed by two observers blinded to the treatment protocol, and the results were averaged. Control experiments in which the primary antibody was not added did not exhibit any positive staining.

Real-time monitoring of bioluminescence. The methods were similar to those previously described (Nakamura et al., 2010). Ex vivo explants of the SCN from young adult (3-6 months) and middle-aged (13-16 months) PER2::LUC mice were prepared between Zeit-

geber time 10-11, with the explants entering the Lumicycle photometer (Actimetris). The bioluminescence signal was counted in $1 \mathrm{~min}$ bins for at least $7 \mathrm{~d}$ without changing media, and data were normalized by subtraction of the $24 \mathrm{~h}$ running average from the raw data and then smoothed with a $2 \mathrm{~h}$ running average (Origin Lab software). The amplitude was summed by the highest point and the lowest point of the each cycle.

Statistics. Statistical significances between two groups were determined by Student's unpaired $t$ test. Statistical significances for three or more groups were determined by one-way ANOVA followed by Tukey's post hoc analyses. All results are presented as the mean \pm SEM and were considered significant at $p<0.05$.

\section{Results}

Reduced circadian behavioral rhythm in middle-aged mice We observed clear reduction in the amplitude of the circadian locomotor rhythm in the 12-14 month, "middle-aged" mice when compared with younger animals (Fig. 1). Both young and middle-aged mice expressed clear rhythms in wheel-running activity in both LD and constant darkness (DD) conditions; however, the amplitudes are markedly different. Along with the reduction in overall activity, middleaged mice exhibited more fragmented activity (young, $3.79 \pm 0.5$ bouts/d; middle aged, $6.28 \pm 0.8 ; p<0.05$ ). Most of the circadian parameters that we could measure continue to decline with age.

\section{Reduced multiunit neural activity rhythms in the SCN and SPZ of middle-aged mice}

We implanted single bipolar electrodes into either the SCN $(n=$ $8)$ or SPZ $(n=10)$ to monitor the long-term multiunit neural activity (MUA) (Fig. 2). All of the young mice $(n=4)$ exhibited clear daily rhythms in MUA in the SCN when they were exposed to LD cycles as well as robust free-running circadian rhythms when were placed in DD. The peak in MUA occurred in the

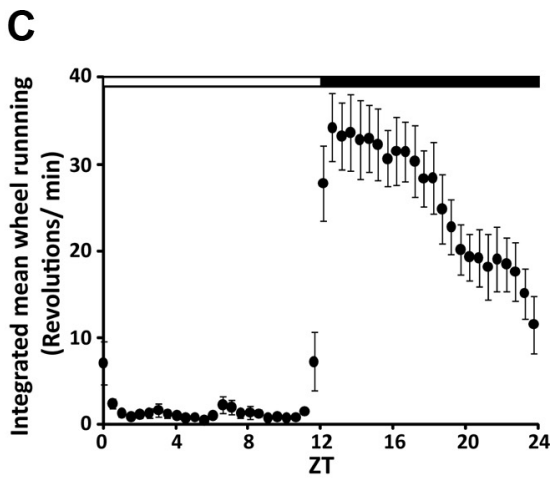

D

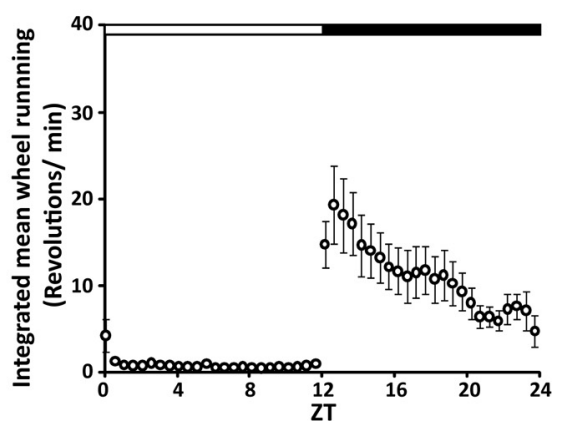

Figure 1. Reduced circadian behavioral rhythm in middle-aged mice. Representative double-plotted actograms of wheelin $(57 \mathrm{BL} / 6 \mathrm{~J}$ mice showing the effect of aging on circadian rhythms of locomotor activity of young $(\boldsymbol{A})$ and at the top of the figure; open bars are light phases and closed are dark. $C, D$, Integrated mean wheel-running activities condition (C: young, $n=8 ;$ : middle-aged, $n=10$ )

middle of the day or subjective day, in approximate antiphase with locomotor activity. In contrast, all of the middle-aged SCN $(n=4)$ showed continually fluctuating levels of MUA leading to degraded "noisy" circadian rhythms on LD cycles and in DD when compared with the records from young animals. The MUA of SCN negatively correlated to the locomotor activity. The correlation coefficients between locomotor activity and MUA in the SCN of young and middle-aged mice were $-0.42 \pm 0.04$ and $-0.30 \pm 0.09$, respectively. The day-night ratio of MUA in the middle-aged SCN (1.50 \pm 0.37 -fold) was significantly lower $(p<0.05)$ compared with the young mice $(3.00 \pm 0.83$-fold). Interestingly, the accumulated MUA count per day in the middle-aged SCN $\left(1.62 \times 10^{6}\right.$ MUA counts/d $)$ was significantly larger $(p<0.05)$ than young SCN $\left(1.15 \times 10^{6}\right.$ MUA counts/d $)$.

The SPZ of young mice $(n=5)$ also showed clear daily and circadian rhythms (Fig. 3). In contrast to the SCN, the SPZ exhibited circadian rhythms with peak activity occurring at night or during the subjective night, closely mirroring locomotor activity. In young adult SPZ, the MUA exhibited clear rhythms with daynight ratio (night/day) reached $3.23 \pm 1.50$-fold. Although the middle-aged SPZ $(n=5)$ exhibited relatively clear MUA rhythm in both LD and DD, the day-night ratio (night/day) was significantly lower $(p<0.05)$ than young SPZ $(1.53 \pm 0.51$-fold $)$. The accumulated MUA in the middle-aged SPZ $\left(2.70 \times 10^{6}\right.$ MUA counts/d) was also larger than young SPZ $\left(1.16 \times 10^{6}\right.$ MUA counts/d), but it did not reach to the significant level $(p=0.08)$. In addition, the MUA of SPZ positively correlated to the locomotor activity, and aging significantly reduced the correlation coefficients $(0.60 \pm 0.03$ for young and $0.36 \pm 0.07$ for middle-aged; $p<0.05)$. 

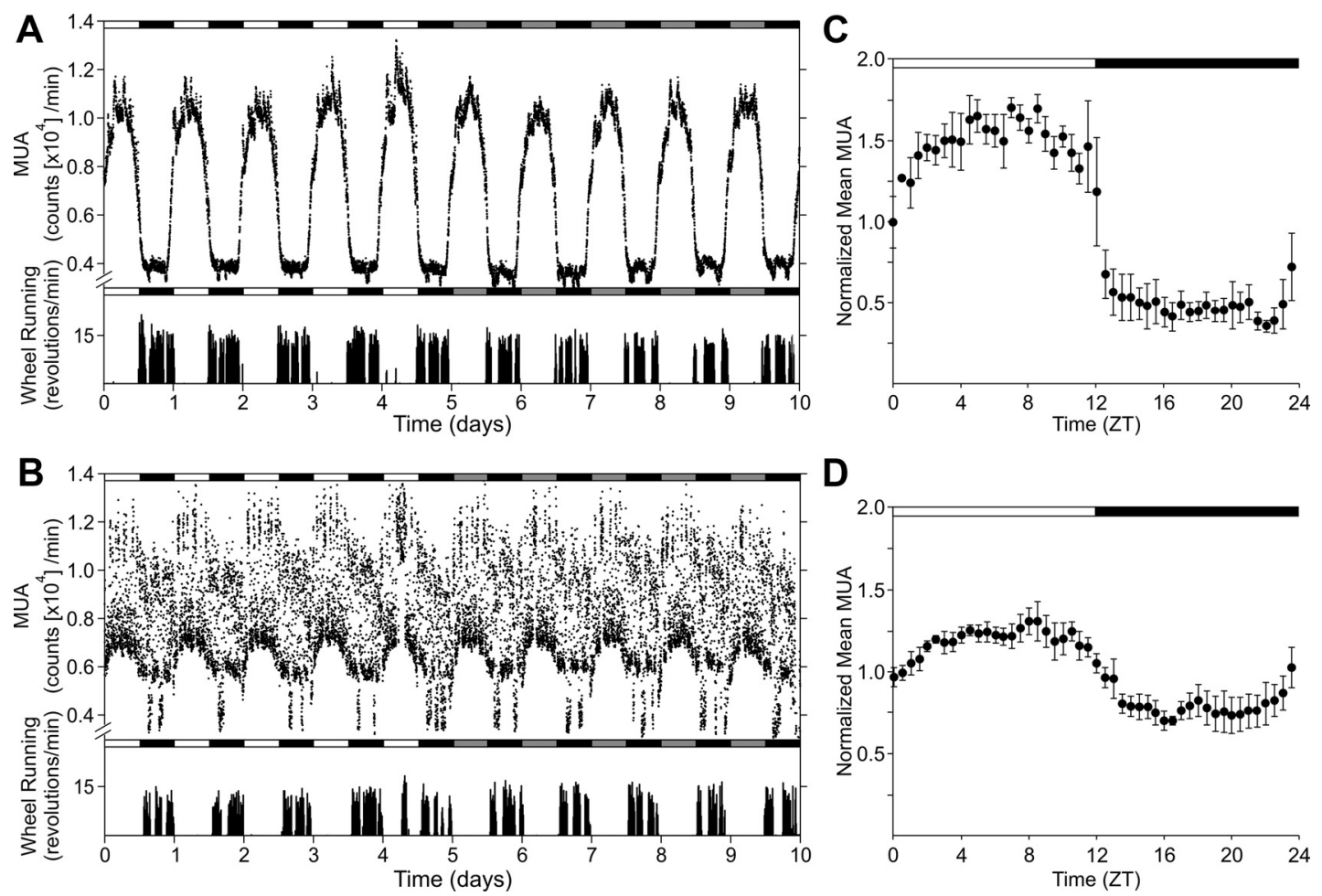

Figure 2. Reduced MUA rhythm in the SCN of middle-aged mice in vivo. Representative serial-plotted actograms of neural and locomotor activity showing diurnal and circadian rhythms of MUA in the SCN of young $(\boldsymbol{A})$ and middle-aged $(\boldsymbol{B})$ mice. Lighting conditions are indicated at the top of the figure; open bars are light phases and closed are dark. Bottom trace represents simultaneous recorded locomotor activity. The number of spikes for MUA or activity counts for locomotor activity was counted every minute. $\boldsymbol{C}, \boldsymbol{D}$, Integrated mean activities were plotted for the LD condition. Each of four individual recordings was normalized with $24 \mathrm{~h}$ moving average and integrated for each group. Data are shown mean \pm SEM, $n=4$ per group.
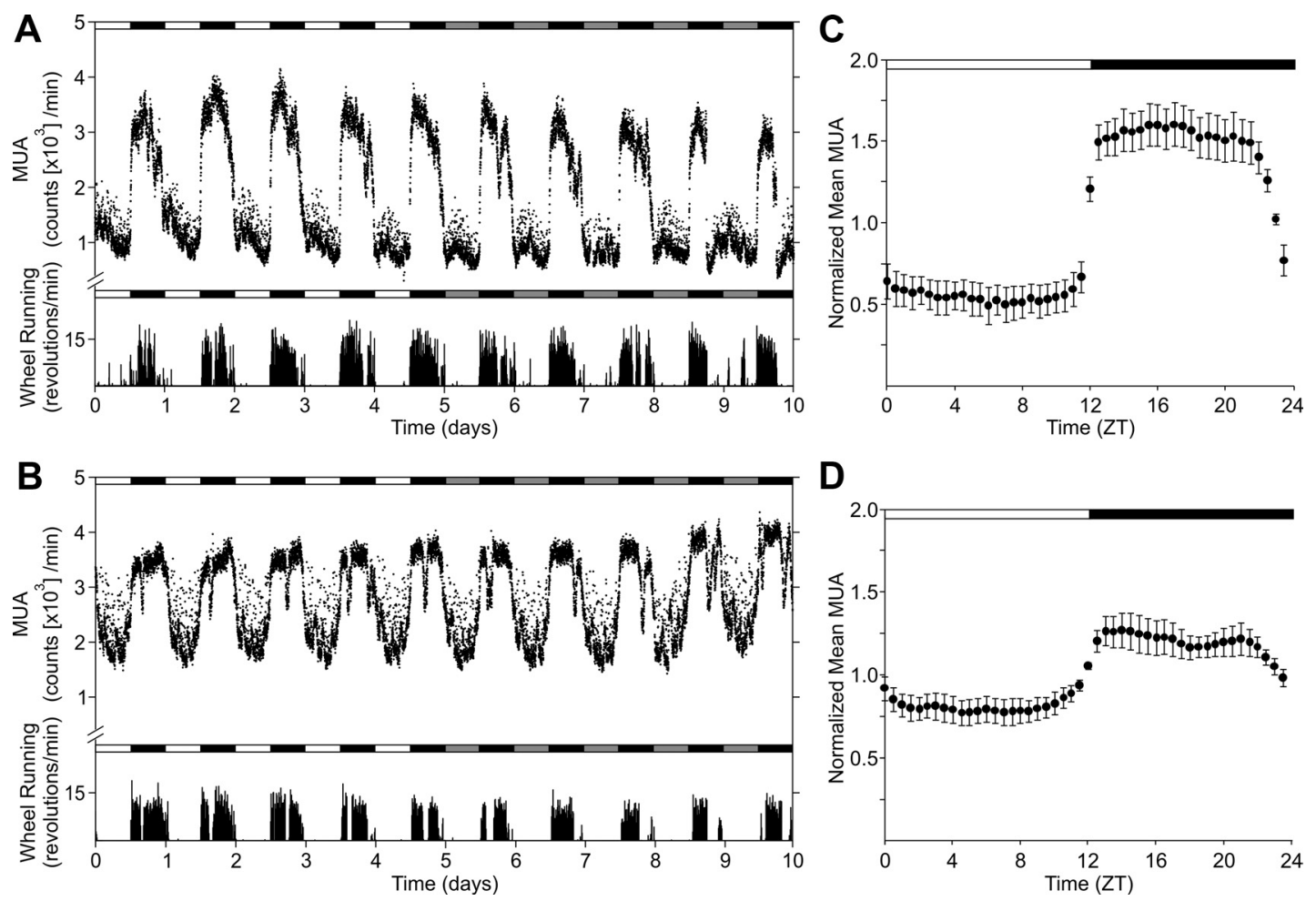

Figure 3. Reduced MUA rhythm in the SPZ of middle-aged mice in vivo. Representative serial-plotted actograms of neural and locomotor activity showing diurnal rhythms of MUA in the SPZ of young $(\boldsymbol{A})$ and middle-aged $(\boldsymbol{B})$ mice. Lighting conditions are indicated at the top of the figure; open bars are light phases and closed are dark. Bottom trace represents simultaneous recorded locomotor activity. The number of spikes for MUA or activity counts for locomotor activity was counted every minute. C, D, Integrated mean activities were plotted for the LD condition. Each of four individual recordings was normalized with $24 \mathrm{~h}$ moving average and integrated for each group. Data are shown mean \pm SEM, $n=5$ per group. 
PER2 expression rhythms of middleaged SCN in vivo and ex vivo

To determine if the molecular machinery necessary to generate circadian oscillations was affected by aging, we used immunohistochemistry (IHC) and bioluminescence to examine PER2 expression rhythms within the SCN (Fig. 4). For IHC experiments, the wheel-running activity of young and middle-aged mice in LD was monitored, and the mice were placed in DD for $3 \mathrm{~d}$. Mice $(n=$ 3-9 per time point) from each age group were sampled at four different points in the daily cycle (CT 2, 8, 14, and 20). PER2 was strongly expressed in the SCN of both ages with immunoreactivity seen throughout the SCN. As measured by the PER2 rhythm, we do not see any evidence that the molecular clockwork is disrupted in the aging mice. We also examined bioluminescence rhythms from young and middle-aged SCN from PER2::LUC knock-in mice to follow the amplitude and phase of each tissue over time. Both young and aged SCN clearly showed circadian rhythms of PER2::LUC expression. While most parameters were the same, there was a significant difference in the amplitudes between young and middleaged group after the first couple of cycles in culture. Although the free-running periods did not differ between the groups, there was also a significant increase in cycle-to-cycle variability between young $(1.17 \pm 0.1)$ and middle-aged mice $(2.74 \pm 0.2 ; p=0.005)$. Overall, the bioluminescence records from the SCN were very similar between the two ages.

\section{Discussion}

The most important observation of the present study is that the amplitudes of electrical activity rhythms recorded from the SCN in vivo are already in decline by middle age (Fig. 2). This observation of reduced circadian output complements prior recordings made in SCN brain slice preparations (Satinoff et al., 1993; Watanabe et al., 1995; Nygard et al., 2005; Biello, 2009), as well as cultured SCN neurons (Aujard et al., 2001) that indicate the in vitro MUA rhythms are reduced in amplitude. The results we have obtained using in vivo recording gives us assurance that the age-related changes observed in the brain slice preparation are due to bona fide alterations in neuronal rhythmicity associated with aging. One apparent difference between our in vivo recording and prior in vitro studies in aged animals is the irregularity of the SCN electrical rhythms. Unlike the electrical rhythms of young animals, middle-aged animals showed high variability between adjacent measurement intervals. Such irregularity is not evident in slice SCN recordings; however, some of the difference with prior studies may be due to their larger collection intervals ( $1 \mathrm{~h}$ vs $1 \mathrm{~min}$ bins used in the present study). In addition, as there is evidence that the SCN is influenced by activity in other brain regions (Deboer et al., 2003; Vansteensel et al., 2003), the increased bin-to-bin variability could reflect degradation of regulation by other brain regions rather than reflecting a noisy aged SCN. Our in vivo data are consistent with an earlier study on glucose utilization comparing young and middle-aged female rats (Wise et al., 1988). Such metabolic differences might be expected if spontaneous electrical activity is reduced in aged animals in vivo. The middle-aged mice showed a reduced correlation between MUA and locomotor activity, a finding that is consistent with a reduced circadian output.

We were also able to extend our analysis to a major output pathway of the SCN, the SPZ. The aged SPZ exhibits a reduced amplitude electrical rhythm that is expressed in antiphase to the SCN (Fig. 3). An obvious interpretation of this result is that the age-related reduction in amplitude of the SCN electrical rhythm leads to a reduced drive on the SPZ. Such an interpretation is consistent with the results from experiments in rat in which when the SCN was surgically isolated, circadian rhythms were lost in regions surrounding the SCN (Inouye and Kawamura, 1979). In this model, the SCN is the source of neural circadian rhythms, and other brain regions are made rhythmic through their connection with the SCN. One has to be cautious about this interpretation, however, as we now recognize that many brain regions exhibit rhythms in molecular expression (Abe et al., 2002), and it is possible that the electrical rhythm in the SPZ, although synchronized by the SCN, is of intrinsic origin. The fact that middle-aged SPZ recordings are more regular (although still less regular than in young animals) than middle-aged SCN rhythms suggests that the SCN may not simply drive a rhythm on the SPZ, at least in its high-frequency detail.

Interacting molecular feedback loops driving rhythmic transcription and translation of key clock genes such as Period (Hastings et al., 2003) are at the core of the oscillatory mechanism responsible for driving circadian oscillations. As a first screen for possible deficits in this molecular clockwork, we looked at PER2 expression (Fig. 4A, B). We did not see any evidence for an agerelated disruption with this in vivo assay. Using ex vivo bioluminescence monitoring of PER2::LUC-driven luciferase activity in transgenic mice, we found both the phase and the amplitude on the first cycle to be indistinguishable between young and middleaged mice (Fig. 4C). There was a significant impact on the amplitude after the third cycle that may indicate an age-related increase in damping of these molecular oscillations. Other studies looking 
at the impact of aging on clock gene expression within the SCN have found robust rhythms in the expression of Period genes (Asai et al., 2001; Yamazaki et al., 2002; Kolker et al. 2004; Davidson et al., 2008) while others have found evidence for an agerelated disruption in Per2 (Weinert et al., 2001) and other clock genes (Kolker et al., 2003; Wyse and Coogan, 2010). Our observation that the electrical rhythms are already disrupted at middle age at a point in which we did not see much evidence for disruption of the PER2 rhythm suggests that circadian MUA output from the SCN may be particularly sensitive to the impact of aging. The weakening of the electrical output of the SCN provides a possible physiological explanation for changes in the phase relationships between the central clock and peripheral organs that are associated with aging in the circadian system (Yamazaki et al., 2002).

This degradation of circadian output at the level of the SCN is likely to have profound consequences on the individual's health (Takahashi et al., 2008). It is becoming increasing clear that robust daily rhythms of sleep/wake are essential to good health. This brings up the question as to the extent that circadian perturbations exacerbate the age-related alterations in the functions of other physiological systems. In humans, a weakening of the circadian system can account for certain aspects of age-related sleep changes, and treating the circadian system in the elderly offers a potential target for ameliorating age-related changes in sleep and daytime alertness (Bliwise, 1993; Cajochen et al., 2006). In the United States, $40-70 \%$ of the elderly population is estimated to experience chronic sleep disturbances (Van Someren, 2000). Prior work on humans found evidence for age-related decline in sleep consolidation and suggests a reduced strength of a circadian signal promoting sleep in the early morning (Czeisler et al., 1999; Dijk et al., 1999). The reduced amplitude of circadian output as measured by electrical rhythm within the rodents SCN may provide the mechanistic underpinning of this reduced circadian signal and may be an important model for understanding similar changes in humans.

\section{References}

Abe M, Herzog ED, Yamazaki S, Straume M, Tei H, Sakaki Y, Menaker M, Block GD (2002) Circadian rhythms in isolated brain regions. J Neurosci 22:350-356.

Asai M, Yoshinobu Y, Kaneko S, Mori A, Nikaido T, Moriya T, Akiyama M, Shibata S (2001) Circadian profile of Per gene mRNA expression in the suprachiasmatic nucleus, paraventricular nucleus, and pineal body of aged rats. J Neurosci Res 66:1133-1139.

Aujard F, Herzog ED, Block GD (2001) Circadian rhythms in firing rate of individual suprachiasmatic nucleus neurons from adult and middle-aged mice. Neurosci 106:255-261.

Biello SM (2009) Circadian clock resetting in the mouse changes with age. Age 31:293-303.

Bliwise DL (1993) Sleep in normal aging and dementia. Sleep 16:40-81.

Cai A, Scarbrough K, Hinkle DA, Wise PM (1997) Fetal grafts containing suprachiasmatic nuclei restore the diurnal rhythm of CRH and POMC mRNA in aging rats. Am J Physiol 273:1764-1770.

Cajochen C, Münch M, Knoblauch V, Blatter K, Wirz-Justice A (2006) Agerelated changes in the circadian and homeostatic regulation of human sleep. Chronobiol Int 23:461-474.

Czeisler CA, Duffy JF, Shanahan TL, Brown EN, Mitchell JF, Rimmer DW, Ronda JM, Silva EJ, Allan JS, Emens JS, Dijk DJ, Kronauer RE (1999) Stability, precision, and near-24-hour period of the human circadian pacemaker. Science 284:2177-2181.

Davidson AJ, Yamazaki S, Arble DM, Menaker M, Block GD (2008) Resetting of central and peripheral circadian oscillators in aged rats. Neurobiol Aging 29:471-477.

Deboer T, Vansteensel MJ, Détári L, Meijer JH (2003) Sleep states alter activity of suprachiasmatic nucleus neurons. Nat Neurosci 6:1086-1090.

Dijk DJ, Duffy JF, Riel E, Shanahan TL, Czeisler CA (1999) Ageing and the circadian and homeostatic regulation of human sleep during forced desynchrony of rest, melatonin and temperature rhythms. J Physiol 516:611-627.
Duncan MJ, Herron JM, Hill SA (2001) Aging selectively suppresses vasoactive intestinal peptide messenger RNA expression in the suprachiasmatic nucleus of the Syrian hamster. Brain Res Mol Brain Res 87:196-203.

Hastings MH, Reddy AB, Maywood ES (2003) A clockwork web: circadian timing in brain and periphery, in health and disease. Nat Rev Neurosci 4:649-661.

Inouye ST, Kawamura H (1979) Persistence of circadian rhythmicity in a mammalian hypothalamic "island" containing the suprachiasmatic nucleus. Proc Natl Acad Sci U S A 76:5962-5966.

Kawakami F, Okamura H, Tamada Y, Maebayashi Y, Fukui K, Ibata Y (1997) Loss of day-night differences in VIP mRNA levels in the suprachiasmatic nucleus of aged rats. Neurosci Lett 222:99-102.

Kolker DE, Fukuyama H, Huang DS, Takahashi JS, Horton TH, Turek FW (2003) Aging alters circadian and light-induced expression of clock genes in golden hamsters. J Biol Rhythms 18:159-169.

Kolker DE, Vitaterna MH, Fruechte EM, Takahashi JS, Turek FW (2004) Effects of age on circadian rhythms are similar in wild-type and heterozygous Clock mutant mice. Neurobiol Aging 25:517-523.

Krajnak K, Kashon ML, Rosewell KL, Wise PM (1998) Aging alters the rhythmic expression of vasoactive intestinal polypeptide mRNA but not arginine vasopressin mRNA in the suprachiasmatic nuclei of female rats. J Neurosci 18:4767-4774.

Kudo T, Schroeder A, Loh DH, Kuljis D, Jordan MC, Roos KP, Colwell CS (2011) Dysfunctions in circadian behavior and physiology in mouse models of Huntington's disease. Exp Neurol 228:80-90.

Nakamura TJ, Sellix MT, Kudo T, Nakao N, Yoshimura T, Ebihara S, Colwell CS, Block GD (2010) Influence of the estrous cycle on clock gene expression in reproductive tissues: effects of fluctuating ovarian steroid hormone levels. Steroids 75:203-212.

Nakamura W, Yamazaki S, Nakamura TJ, Shirakawa T, Block GD, Takumi T (2008) In vivo monitoring of circadian timing in freely moving mice. Curr Biol 18:381-385.

Nygård M, Hill RH, Wikström MA, Kristensson K (2005) Age-related changes in electrophysiological properties of the mouse suprachiasmatic nucleus in vitro. Brain Res Bull 65:149-154.

Satinoff E, Li H, Tcheng TK, Liu C, McArthur AJ, Medanic M, Gillette MU (1993) Do the suprachiasmatic nuclei oscillate in old rats as they do in young ones? Am J Physiol 265:R1216-R1222.

Takahashi JS, Hong HK, Ko CH, McDearmon EL (2008) The genetics of mammalian circadian order and disorder: implications for physiology and disease. Nat Rev Genet 9:764-775.

Turek FW, Penev P, Zhang Y, van Reeth O, Zee P (1995) Effects of age on the circadian system. Neurosci Biobehav Rev 19:53-58.

Van Reeth O, Zhang Y, Zee PC, Turek FW (1994) Grafting fetal suprachiasmatic nuclei in the hypothalamus of old hamsters restores responsiveness of the circadian clock to a phase shifting stimulus. Brain Res 643:338-342.

Van Someren EJ (2000) Circadian rhythms and sleep in human aging. Chronobiol Int 17:233-243.

Vansteensel MJ, Yamazaki S, Albus H, Deboer T, Block GD, Meijer JH (2003) Dissociation between circadian Perl and neuronal and behavioral rhythms following a shifted environmental cycle. Curr Biol 13:1538-1542.

Viswanathan N, Davis FC (1995) Suprachiasmatic nucleus grafts restore circadian function in aged hamsters. Brain Res 686:10-16.

Watanabe A, Shibata S, Watanabe S (1995) Circadian rhythm of spontaneous neuronal activity in the suprachiasmatic nucleus of old hamster in vitro. Brain Res 695:237-239.

Weinert H, Weinert D, Schurov I, Maywood ES, Hastings MH (2001) Impaired expression of the mPer2 circadian clock gene in the suprachiasmatic nuclei of aging mice. Chronobiol Int 18:559-565.

Wise PM, Cohen IR, Weiland NG, London ED (1988) Aging alters the circadian rhythm of glucose utilization in the suprachiasmatic nucleus. Proc Natl Acad Sci U S A 85:5305-5309.

Wyse CA, Coogan AN (2010) Impact of aging on diurnal expression patterns of CLOCK and BMAL1 in the mouse brain. Brain Res 1337:21-31.

Yamazaki S, Straume M, Tei H, Sakaki Y, Menaker M, Block GD (2002) Effects of aging on central and peripheral mammalian clocks. Proc Natl Acad Sci U S A 99:10801-10806.

Yoo SH, Yamazaki S, Lowrey PL, Shimomura K, Ko CH, Buhr ED, Siepka SM, Hong HK, Oh WJ, Yoo OJ, Menaker M, Takahashi JS (2004) PERIOD2:: LUCIFERASE real-time reporting of circadian dynamics reveals persistent circadian oscillations in mouse peripheral tissues. Proc Natl Acad Sci U S A 101:5339-5346. 